\title{
The influence of temperature on viscoelastic friction properties
}

\author{
C. Putignano ${ }^{\mathrm{a}, *}$, T. Reddyhoffa ${ }^{\mathrm{a}}$, D. Dini ${ }^{\mathrm{a}}$ \\ ${ }^{a}$ Department of Mechanical Engineering, Imperial College London, Exhibition Road, \\ South Kensington, London,SW7 2AZ, United Kingdom.
}

\begin{abstract}
Viscoelastic friction strongly depends on temperature, which determines the material stiffness and, therefore, given a constant load, the volume that is deformed and dissipates energy. We compare the results obtained by a numerical approach introduced in Ref. [1] with measurements that separate viscoelastic losses from Coulomb contribution. This is done for a range of temperatures. We show that viscoelastic friction curves for different temperatures can be arranged into a single master curve using a frequency shift coefficient, which can be found from the characterization of the viscoelastic material response. This shows that it is possible to accurately a) use dynamic material analysis data to extrapolate viscoelastic friction measurements to values outside the tested range, and b) use a tribometer to obtain fundamental viscoelastic material properties.
\end{abstract}

Keywords: viscoelastic contact mechanics, viscoelastic friction, contact mechanics measures.

\section{Introduction}

Viscoelastic friction is an issue that is currently the subject of significant attention from engineering and tribology researchers. In order to obtain a clearer understanding of this phenomenon, a large variety of experimental techniques and numerical models have been developed ([8], [9], [10], [11], [14], [15], [16], [1] ). This level of interest is primarily due to the large number

\footnotetext{
*Corresponding author. Phone: +44 (0)2075947242

Email address: c.putignano12@imperial.ac.uk (C. Putignano)
} 
of polymer and rubber-based engineering components in which viscoelastic dissipation constitutes one of the main sources of mechanical losses. Classical examples include tires and seals $([2],[3])$, but innovative applications in fields such as bioengineering [4], nanotechnology [5] and "green" technology [6] are now also requiring an improved understanding of viscoelastic friction mechanisms. Tackling these problems is, however, very challenging for a number of reasons. Firstly, any numerical, analytical or experimental approach must incorporate the effects induced by the presence of the surface roughness, whose power spectrum can extend over several orders of magnitude [7]. Furthermore, due to the particular nature of this class of materials, not only time-dependent effects, but also the strong dependence of the material properties on the temperature must be accounted for. Indeed, varying the temperature dramatically changes the material stiffness and, consequently, the deformed volume and the amount of energy dissipated ([26],[27]).

Given the intricacy of the problem, analytical approaches have proved challenging. A pioneering attempt was made by Hunter [8] for the case of two-dimensional (2D) contacts, i.e. for a rigid cylinder in contact with a viscoelastic half-space. Although many other contributions have later followed the same approach [32], this model has a major limitation in that it can handle only the simplest form of viscoelasticity, i.e. materials with only a single relaxation time. Unfortunately, as it has been shown consistently in the literature $([1],[17],[31],[27])$, real viscoelastic materials are characterized by complex mechanical behaviour with a large spectrum of relaxations times. In the last fifteen years, Persson ([11], [12]) has developed a new theory that can be applied - in first approximation - to a general viscoelastic material. Recently, he has also investigated the role of temperature ([13],[26]), but, to the best of authors' knowledge, no definitive quantitative answer to the problem has been provided.

Several numerical techniques have been implemented to overcome these limitations. On the one hand, there are Finite Element Methods (FEM) $([15,18,19,20,21])$ that can simulate real viscoelastic materials with many relaxation times and complex geometrical configurations, but have been conceived mainly for structural modelling so that, especially when the roughness of the contacting bodies is accounted for and high resolution at asperity level is required, it may be difficult to obtain accurate solutions for contact problems. On the other hand, Boundary Elements (BE) techniques provide good solutions in terms of stresses and strains $([1],[7],[33],[35])$, but, since they are based on the superposition principle and, consequently, require translational 
invariance and system linearity to be satisfied, may suffer from limitations linked to the generality of the contact domain and material properties. In particular, one of the main issues relates to the temperature fields; specifically, the presence of temperature gradients implies varying material properties along the contact region and, consequently, hinders the applicability of the methodology.

Experimental investigations have also shown that temperature effects can have a dramatic influence on the contact properties and can affect the reliability of the models developed under isothermal assumptions ([26],[27]). Interestingly, this issue was first acknowledged by Grosch who presented an investigation into the dependence of rubber friction on sliding speed and temperature. In spite of an experimental setup [10] that did not separate the different forms of dissipation, i.e. Coulomb and viscoelastic friction, Grosch was able to show a close relation between friction, material properties and temperature.

This paper aims to shed light on the interdependence between viscoelastic losses and thermal effects by carrying out friction measurements at different temperatures with a new setup that, unlike Grosch's, is capable of separating the Coulomb contribution from the viscoelastic dissipation. This enables us to compare the measured viscoelastic friction with the numerical predictions obtained by employing the mathematical formulation in Ref. [1]. The primary purpose of this experimental campaign is to validate the numerical method still in isothermal conditions, but over a large temperature range. Furthermore, by investigating the relation between friction and material properties, we propose a methodology to build a master curve for the prediction of frictional response extrapolating data at different temperatures. The paper is set out as follows. Section 2 briefly outlines the viscoelastic model - including the time-temperature superposition effect - and the mathematical formulation employed for the numerical contact predictions. Section 3 describes the experimental procedure employed to measure the friction and to separate Coulomb and viscoelastic friction. Finally, in Section 4, the experimental outcomes, compared with the numerical predictions, are shown and discussed. The last Section includes conclusions and closing remarks. 


\section{Material Constitutive Model and Numerical Formulation}

\subsection{Linear Viscoelastic Model}

The viscoelastic response of linear rubber-like materials can be modelled by the following integral equation :

$$
\varepsilon(t)=\int_{-\infty}^{t} d \tau \mathcal{J}(t-\tau) \dot{\sigma}(\tau)
$$

where $\varepsilon(t)$ is the time-dependent strain, $\sigma(t)$ is the stress (the symbol '.' stands for the time derivative), and the function $\mathcal{J}(t)$ is the creep function that must satisfy causality, i.e. $\mathcal{J}(t<0)=0$. The most general form of $\mathcal{J}(t)$ is given by [17]:

$$
\mathcal{J}(t)=\mathcal{H}(t)\left[\frac{1}{E_{0}}-\int_{0}^{+\infty} d \tau \mathcal{C}(\tau) \exp (-t / \tau)\right],
$$

where $\mathcal{H}(t)$ is the Heaviside step function, the real quantity $E_{0}$ is the rubber elastic modulus of the material at zero-frequency, $\mathcal{C}(\tau)$ is a strictly positive function defined as creep spectrum( [17] [29]), and $\tau$ is the relaxation time, continuously distributed on the real axis.

Now, we observe that Eq. (2) can be Fourier transformed as $\varepsilon(\omega)=$ $\sigma(\omega) / E(\omega)$ where $E(\omega)$ is the viscoelastic modulus equal to $E(\omega)=[i \omega J(\omega)]^{-1}$. Given such a definition, we can then write the following relation for $E(\omega)$ :

$$
\frac{1}{E(\omega)}=\frac{1}{E_{0}}-\int_{0}^{\infty} d \tau \frac{i \omega \tau \mathcal{C}(\tau)}{1+i \omega \tau}=\frac{1}{E_{\infty}}+\int_{0}^{\infty} d \tau \frac{\mathcal{C}(\tau)}{1+i \omega \tau}
$$

From a physical point of view, we observe that, at low frequencies, the material behaves elastically and, more specifically, is in the 'rubbery' region since, for $\omega->0, E(\omega)->E_{0}$. On the other hand, at very high frequency, the material is again elastic but very stiff (brittle-like) and $E(\omega)->E_{\infty}$. In the intermediate frequency range (the so called 'transition' region), the ratio $\operatorname{Im} E(\omega) / \operatorname{Re} E(\omega)$, i.e. the loss tangent, is very large and this is responsible for the viscoelastic energy dissipation.

Temperature has a dramatic influence on such a behavior: a temperature variation produces a shift of the viscoelastic frequency response (plotted against log frequency) along to the frequency axis. Indeed, increasing the temperature entails shifting to the right the viscoelastic modulus since, thanks to the thermal energy, the material tends to relax (see Fig. 1). For 


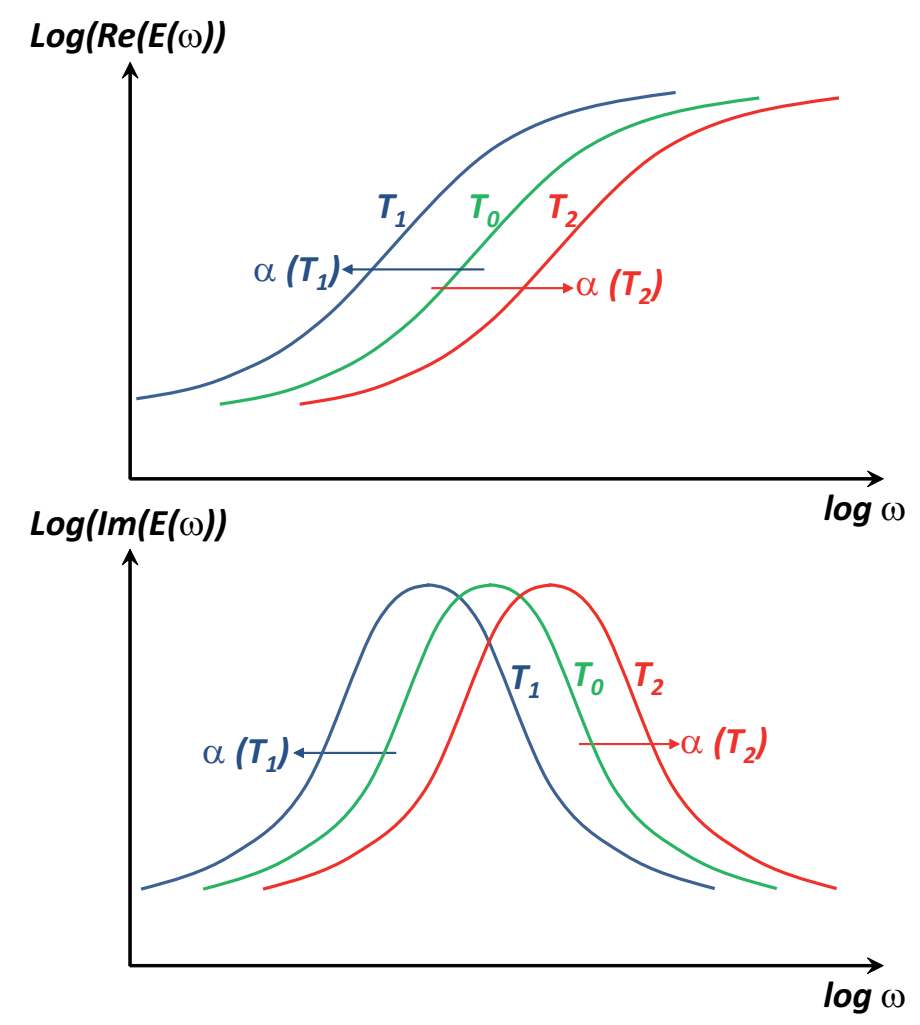

Figure 1: The real $E_{1}=\operatorname{Re}[E(\omega)]$ and the imaginary $E_{2}=\operatorname{Im}[E(\omega)]$ parts of the viscoelastic modulus $E(\omega)$ of a typical rubber-like material for different temperatures.

the majority of linear viscoelastic materials, and in particular for the almost totality of rubber-like materials, a very simple logarithmic relation between temperature and time can be assumed. Therefore, after defining a fixed reference temperature, $T_{0}$, a time-temperature shift factor $\alpha(T)$ can be introduced such that:

$$
E(\omega, T)=E\left(\alpha \omega, T_{0}\right)
$$

The coefficient $\alpha$ can be calculated by means of many empirical expression - the most popular probably being the one suggested by Williams, Landel, and Ferry in Ref. [43] - or can be directly measured by acquiring, at different temperatures, the mechanical response in a given frequency range by employing a Dynamic Mechanical Analysis [17]. 
The frequency-temperature superposition effect has a prominent importance in the characterization of the viscoelastic frequency response $([17],[29],[43])$. Indeed, by means of several isothermal tests, each carried out in a narrow spectral window $\left[\omega_{1}, \omega_{2}\right]$, this principle enables to characterize the viscoelastic modulus over a much broader frequency range. In detail, each frequency curve, acquired at a given temperature $T$, is shifted with $\alpha(T)$ in such a way that a final master curve can be built up. A reference temperature $T_{0}$ is taken to set the frequency scale (the curve at that temperature undergoes no shift).

\subsection{Mathematical formulation of the Contact problem}

In order to approach numerically the contact problem between linear viscoelastic solids, we have to discretize Eq. (2) by assuming $\mathcal{C}(\tau)=\sum_{k} C_{k} \delta\left(\tau-\tau_{k}\right)$ and, therefore, rewriting $\mathcal{J}(t)$ as:

$$
\mathcal{J}(t)=\mathcal{H}(t)\left[\frac{1}{E_{0}}-\sum_{k=1}^{n} C_{k} \exp \left(-t / \tau_{k}\right)\right] .
$$

Now, by moving from the Green function approach already developed in ( [39] [40] [41]) and employing the viscoelasticity model presented above, the boundary element approach developed in Ref. [1] is capable of assessing the mechanics of sliding and rolling viscoelastic contacts. Basically, if we recall the translational invariance and the elastic-viscoelastic correspondence principle [17], the general linear-viscoelastic contact problem between a rigid indenter and a viscoelastic slab can be formulated as:

$$
u(\mathbf{x}, t)=\int_{-\infty}^{t} d \tau \int d^{2} x \mathcal{J}(t-\tau) G\left(\mathbf{x}-\mathbf{x}^{\prime}\right) \dot{\sigma}\left(\mathbf{x}^{\prime}, \tau\right),
$$

where $\mathbf{x}$ is the in-plane position vector, $t$ is the time, $u(\mathbf{x}, t)$ is the normal surface displacement of the viscoelastic solid, $\sigma(\mathbf{x}, t)$ is the normal interfacial stress, $\mathcal{J}(t)$ is given in Eq. (2), and the quantity $G(\mathbf{x})$ is the Green's function. Assuming steady-state conditions, i.e. sliding/rolling motion at constant velocity $\mathbf{v}$, Eq. 6 can be re-written in the form:

$$
u(\mathbf{x}, \mathbf{v})=\int d^{2} x^{\prime} \mathcal{G}\left(\mathbf{x}-\mathbf{x}^{\prime}, \mathbf{v}\right) \sigma\left(\mathbf{x}^{\prime}\right)
$$

where the kernel $\mathcal{G}(\mathbf{x}, \mathbf{v})$ depends parametrically on the speed $\mathbf{v}$ and has the following form: 


$$
\begin{aligned}
\mathcal{G}(\mathbf{x}, \mathbf{v}) & =-\frac{1-\nu^{2}}{\pi}\left\{\frac{1}{E_{\infty}} \frac{1}{|\mathbf{x}|}\right. \\
& \left.+\int_{0}^{+\infty} d \tau C(\tau) \int_{0^{+}}^{+\infty} d z \frac{1}{|\mathbf{x}+\mathbf{v} \tau z|} \exp (-z)\right\} .
\end{aligned}
$$

Such a formulation, based on the isothermal assumption, requires the viscoelastic modulus $E(\omega)$ corresponding to the temperature $T$ of the viscoelastic solid. Furthermore, since the formulation is time-independent, the contact problem can be solved by employing the same approach already developed for the linear elastic rough contact problem studied in ([39], [40], [41]). For further details regarding the methodology, the reader can refer to Ref. [1].

\section{Experimental methodologies}

In this study, friction measurements are made by means of a mini-traction machine, or MTM, (PCS Instruments Ltd, Acton, UK), in which the ball is loaded and rotated against the rotating flat disc (see Fig. 2). It is important that each component is driven by a separate motor, thus enabling any sliding/rolling speed combination (slide/roll ratios).

In these tests, the disc consists of a rubber layer attached to a steel substrate. The thickness of the rubber sheet is chosen to be much larger than the contact area for the given operating conditions so that it can be considered a half-space in the numerical calculations. Furthermore, as shown in Fig. 2, some mineral oil is introduced into the MTM specimen holding unit for temperature control purposes; with particular care having been taken to ensure that the fluid does not reach the contact interface, which remains dry. The presence of the fluid allows the temperature of the viscoelastic body to be adjusted while, at the same time, maintaining isothermal conditions.

For the ball sample, we use PTFE to minimize interfacial losses, here approximated by Coulomb friction, and hence ensure an accurate measure of the viscoelastic losses. This is important not only to reduce this additional source of dissipation, but also to limit local thermal effects and wear, which may affect the reliability of the measurements [1] .

Starting from the approach developed in Ref. [27], we want to separate the viscoelastic losses from interfacial friction components. To this end, it 


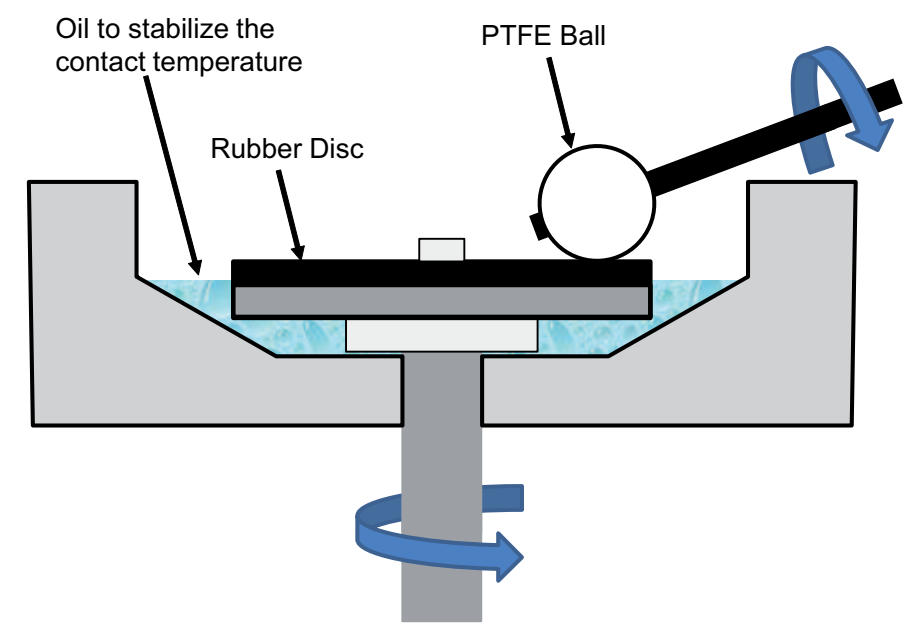

Figure 2: Schematic of the setup for the MTM rig.

is important to note that the Coulomb interfacial friction force, $F_{c}$, strictly depends on the sign of the relative speed of the the ball and the disk $w=$ $u_{\text {disk }}-u_{\text {ball }}: F_{c}$ always changes its sign in accordance to the sign of $w$. The viscoelastic frictional force, $F_{v}$, on the other hand, opposes the speed at which the viscoelastic body is deformed, i.e. $u_{\text {disk }}$. Consequently, if we fix $\left|u_{d i s k}\right|$ and $|w|$, the force on the the load cell attached to the ball drive shaft will be described by the the following four relationships between the sliding conditions and the friction components (see Figure 3):

$$
\begin{aligned}
& u_{\text {ball }}=-\left|u_{\text {ball }}\right| \text { and } w=-|w|: \quad F_{I}=O+F_{c}-F_{v} \\
& u_{\text {ball }}=-\left|u_{\text {ball }}\right| \text { and } w=|w|: \quad F_{I I}=O-F_{c}-F_{v} \\
& u_{\text {ball }}=\left|u_{\text {ball }}\right| \text { and } w=-|w|: \quad F_{I I I}=O-F_{c}+F_{v} \\
& u_{\text {ball }}=\left|u_{\text {ball }}\right| \text { and } w=|w|: \quad F_{I V}=O+F_{c}+F_{v}
\end{aligned}
$$

where $O$ is the transducer's offset (i.e. zero force reading). Therefore, the Coulomb friction force is $F_{c}=\left(F_{I}+F_{I V}-F_{I I}-F_{I I I}\right) / 4$, whereas the viscoelastic friction is $F_{v}=\left(F_{I I I}+F_{I V}-F_{I I}-F_{I I I}\right) / 4$. We process the measurements in this way, so that we can eliminate the contribution of interfacial friction to obtain the components of frictional losses linked solely to the viscoelastic contribution. 


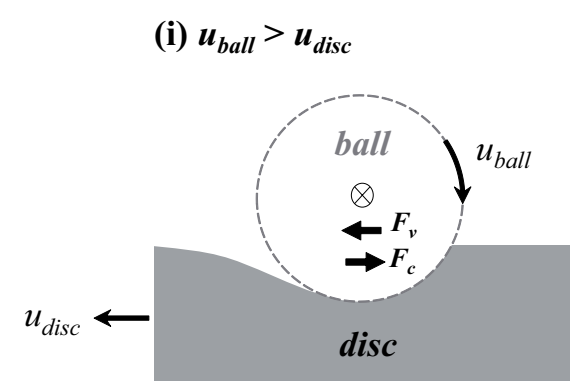

(iii) $\boldsymbol{u}_{\text {ball }}>\boldsymbol{u}_{\text {disc }}$

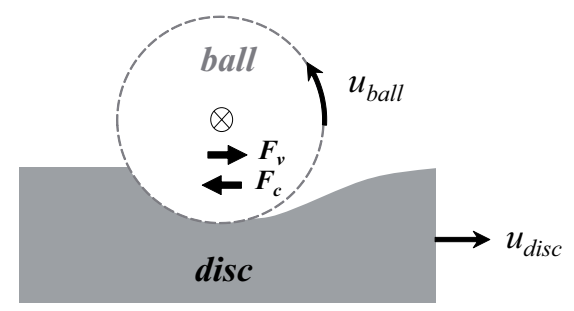

(ii) $\boldsymbol{u}_{\text {ball }}<\boldsymbol{u}_{\text {disc }}$

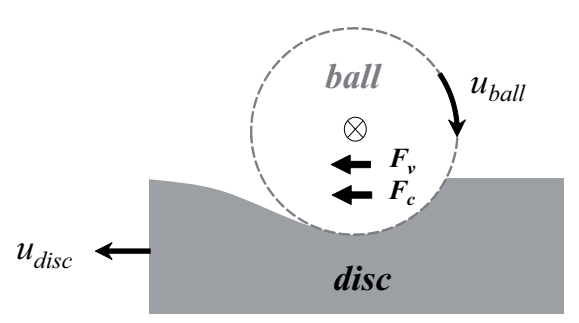

(iv) $\boldsymbol{u}_{\text {ball }}<\boldsymbol{u}_{\text {disc }}$

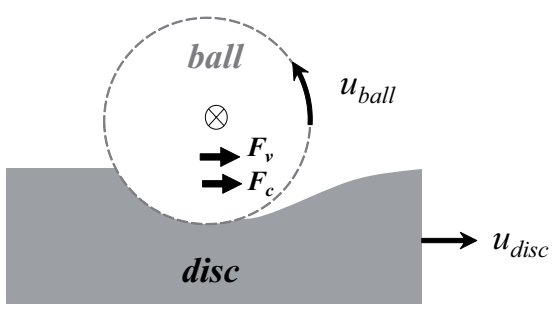

Figure 3: Schematic of the four steps implemented to separate the viscoelastic friction force $F_{v}$ and the Coulomb force $F_{c}$. The arrows refer to the forces on the ball. 


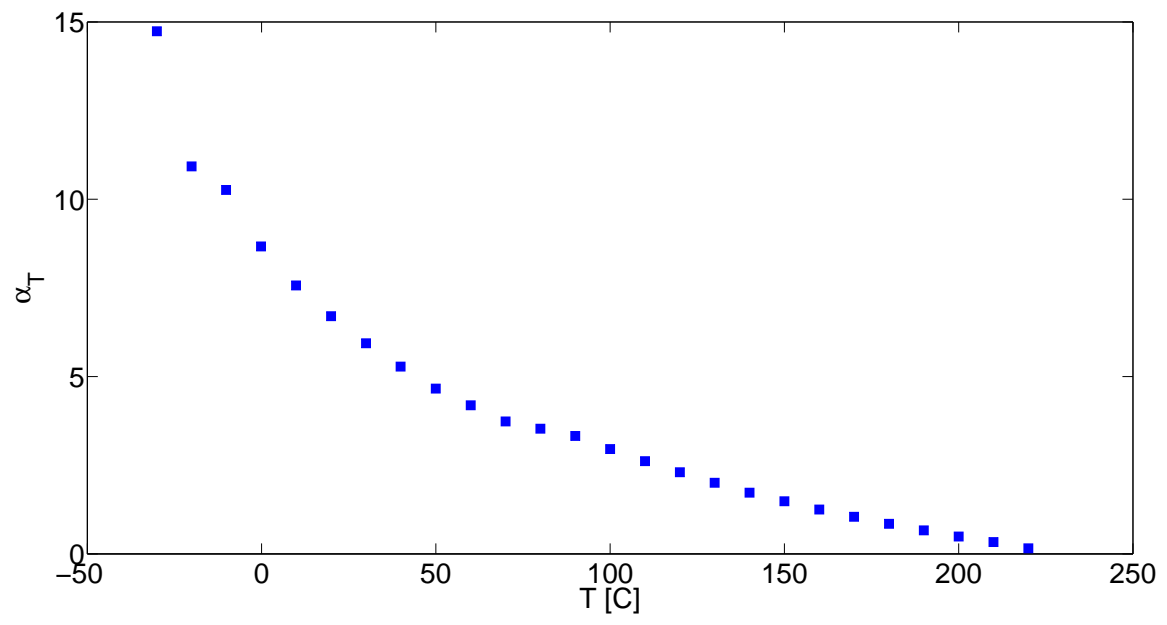

Figure 4: The viscoelastic modulus shift coefficient $\alpha_{T}$ is plotted as a function of the temperature.

\section{Results and discussion}

\subsection{Material Mechanical Response}

Since the aim is to characterize the viscoelastic friction, a highly dissipative polymer, namely Viton fluoroelastomer, is used. The viscoelastic frequency response of this material is characterized by means of a Q800 Dynamic Mechanical Analyzer (DMA) manufactured by TA instruments. This test, carried out in tensile conditions, provides data over a set interval of frequencies and temperatures. The characterization is performed on rubber strips, having a cross section of $2 \mathrm{~mm} \times 5.5 \mathrm{~mm}$ and a length of $35 \mathrm{~mm}$, over a frequency interval between 0.1 and $10 \mathrm{~Hz}$ and temperature varying from -30 to 220 degrees Celsius with $10^{\circ} \mathrm{C}$ step.

With regards to the data post-processing, aimed at building up the master curve and described in the previous Section, the first step requires the calculation of the shifting coefficient $\alpha$ defined in Eq. 6. In Figure 4, we show $\alpha$ as a function of the temperature $T$.

Once the shifting parameters $\alpha$ have been determined, it is possible to build up a master curve over a much extended frequency range. This is what is shown in Figure 5, where the experimental data are plotted in blue - the squares and the circles refer respectively to the imaginary and the real part of 


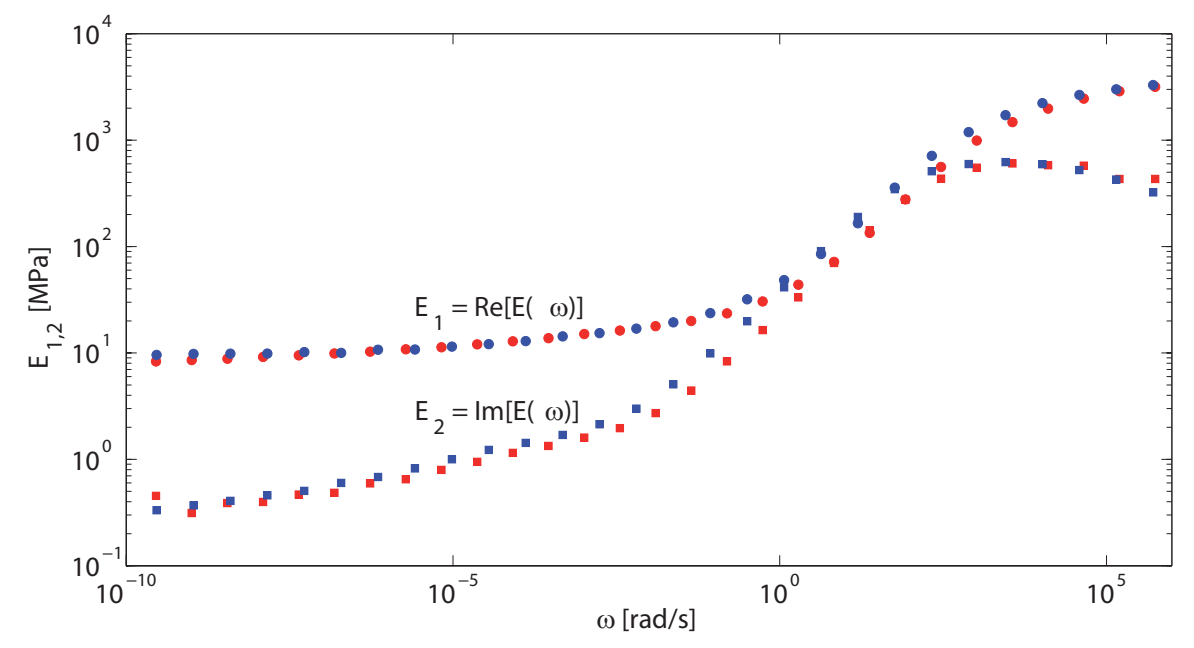

Figure 5: The real part $E_{1}=\operatorname{Re}[E(\omega)]$ (squares) and the imaginary part $E_{2}=\operatorname{Im}$ (circles) of the complex modulus $E(\omega)$ at 30 degrees Celsius. The blue colour refers to the measured values, the red is used for the fit obtained by using Eq. (5).

the viscoelastic modulus. In the same plot, we fit the experimental data using Eq. (5) with a spectrum of relaxation times being assumed to be in geometric progression with Euler's number as common ratio, i.e. $\tau_{k+1} / \tau_{k}=e$. A good agreement, over the entire frequency range, that is extended for more than 15 orders of magnitude, is found between the experiment and the theoretical fitting.

\subsection{Friction measure}

The friction measurements are carried out by using the MTM and following the experimental procedure explained in the previous Section. Tests are conducted at a fixed normal load $F_{N}=25 \mathrm{~N}$ in a speed interval ranging from 20 to $200 \mathrm{~mm} / \mathrm{s}$, and we calculate, for each speed value, the viscoelastic friction coefficient $\mu$, that is obtained by averaging 8 data points. The test is carried out at three different temperature values: $T_{1}=30 \mathrm{C}, T_{2}=60 \mathrm{C}$, $T_{3}=90 \mathrm{C}$.

Figure 6 shows the experimental data with the relative scatter, which is always less than $10 \%$, and the numerical predictions. The two sets of results are in very good agreement with each other over the entire temperature range considered in the study. Incidentally, it can be observed that the 


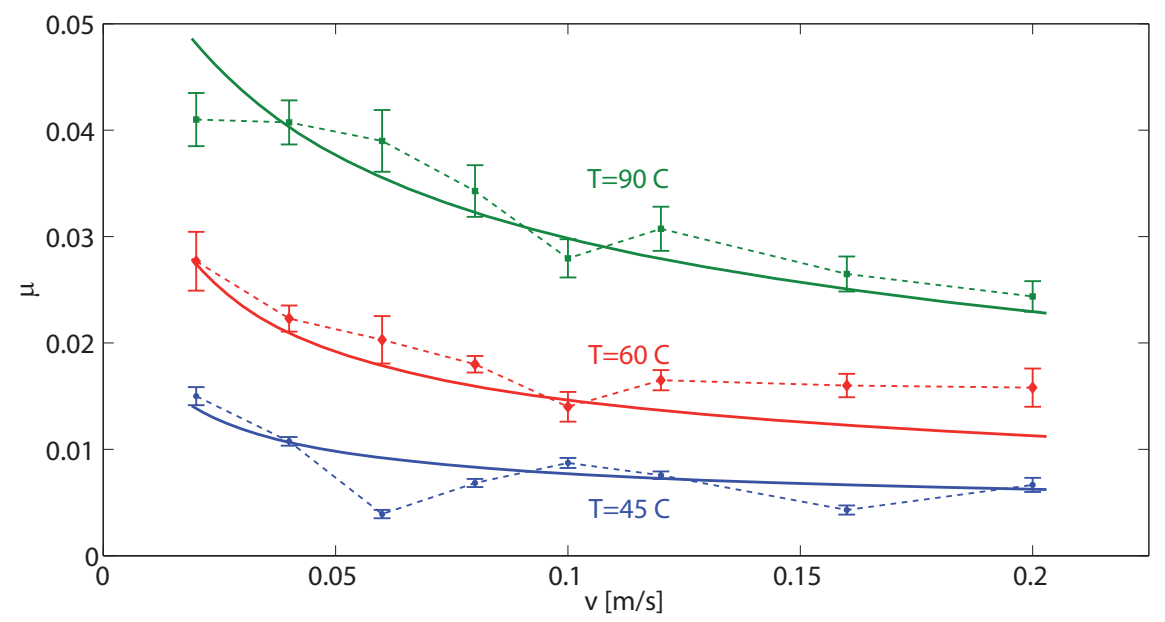

Figure 6: Viscoelastic friction as a function of the rolling speed. Points refer to the experimental results (each solid line show the scatter), blue line to the numerical predictions.

friction coefficient $\mu$ decreases with speed; indeed, the speed range in Fig. 6 corresponds to a frequency interval where the loss modulus of our material is decreasing. Furthermore, it can be noticed that in these temperature and speed ranges, heating effects due to the sliding friction seem not to have a discernible effect on the phenomenon under investigation.

The experimental technique adopted in this paper has the peculiar advantage of enabling a direct comparison with numerical predictions, over a large range of speeds. This is not possible when employing other experimental procedures [10] [36],[1],[25],[9],[37] that do not separate the viscoelastic dissipation from other frictional losses.

After characterizing the friction in a certain speed interval for different temperatures, the question arises as to whether it is possible to extrapolate information over a wider interval, as in the case of the viscoelastic modulus. Indeed, from a physical point of view, viscoelastic losses are strictly related to $E(\omega)$; qualitatively speaking, the storage part determines the volume of material that is deformed and the loss modulus governs the amount of dissipated energy. To test this hypothesis, Fig. 7, shows the experimental data at different temperatures, shifted using the same viscoelastic modulus coefficient $\alpha$ that was plotted in Figure 4. Figure 7 also shows numerical calculations directly computed in the entire range. It can be seen that there 


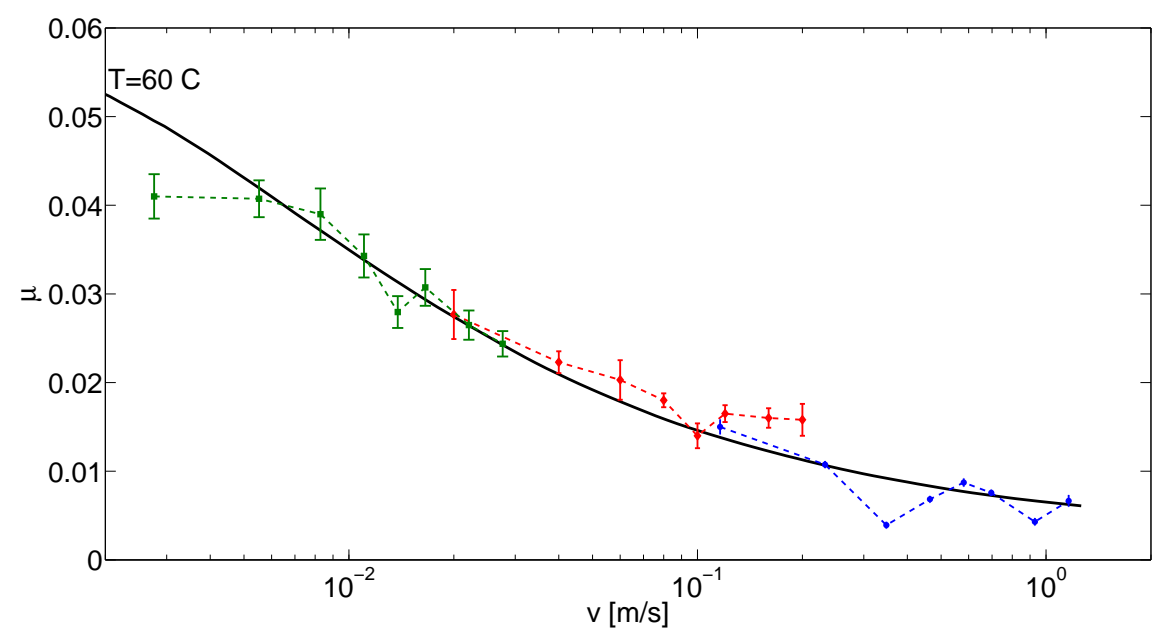

Figure 7: Viscoelastic friction as a function of the rolling speed. Points refer to the experimental results shifted with $\alpha_{T}$, blue line to the numerical predictions.

is again good agreement between both numerical and experimental results.

This confirms the close relationship between modulus and friction. Indeed, it shows that the shifting coefficient $\alpha$ marking the viscoelastic modulus also characterizes the friction coefficient $\mu$. On the one hand, this provides a powerful tool to extrapolate information on the friction coefficient: given a speed $v$ and a temperature $T$, it is possible to obtain the friction coefficient $\mu(v, T)$ carrying out the test at a speed $\alpha v$ and a temperature $T^{\prime}$ :

$$
\mu\left(v, T^{\prime}\right)=\mu\left(\alpha v, T_{0}\right)
$$

Furthermore, Fig. 7 reveals that the coefficient spectrum $\alpha(T)$ can be effectively measured using viscoelastic friction tests. This demonstrates that a tribological measurement of the friction coefficient may provide important support for the mechanical characterization of a viscoelastic material.

\section{Conclusion}

In this contribution we have performed detailed studies to shed light how viscoelastic friction depends on temperature. To do this, an experimental procedure that is capable of isolating the viscoelastic friction component and providing stable measurements over a wide range of speeds and temperatures 
has been developed. This elimination of interfacial frictional losses is crucial in order to validate numerical predictions that relate only to viscoelastic contributions. Indeed, the experimental procedure has proved effective in validating numerical simulations, obtained with the BE methodology developed in Ref. [1]. Interestingly, a decreasing trend of the viscoelastic friction has been shown in the speed range analyzed, which corresponds to a frequency interval where the loss modulus decreases. This trend, which cannot be captured using other experimental methods, has important implications for practical applications, such as tires, where viscoelastic dissipation is a crucial quantity to control.

This work also demonstrates an important correlation between viscoelastic friction and the material mechanical behaviour. We have clearly shown that the viscoelastic modulus shift coefficients $\alpha(T)$ can also be used to shift the frictional response. This enables us to extrapolate the friction over a larger range of speeds than would otherwise be allowed by the tribometer. At the same time, this correspondence may offer an opportunity for the mechanical characterization of viscoelastic materials using frictional measurements alone.

\section{Acknowledgements}

CP gratefully acknowledges the support of Marie Curie Intra-European Fellowship SOFT-MECH (Grant No. 622632). 
[1] G. Carbone, C. Putignano, A novel methodology to predict sliding/rolling friction in viscoelastic materials: theory and experiments., Journal of the Mechanics and Physics of Solids, 61 (8), pp. 1822-1834, (2013).

[2] Bottiglione F., Carbone G., Mangialardi L., Mantriota G., Leakage Mechanism in Flat Seals, Journal of Applied Physics 106 (10), 104902, (2009).

[3] O'Boy D.J., Dowling A.P., Tyre/road interaction noise-A 3D viscoelastic multilayer model of a tyre belt, Journal of Sound and Vibration, $322,4-5,829-850,(2009)$.

[4] D. Craiem, R. L. Magin, Fractional order models of viscoelasticity as an alternative in the analysis of red blood cell (RBC) membrane mechanics, Phys. Biol. 7 013001, (2010).

[5] O. Regeva, S. Vandebrilb, E. Zussmana, C. Clasenb, The role of interfacial viscoelasticity in the stabilization of an electrospun jet, Polymer, 51, 12, 2611-2620, (2010).

[6] T. Tsujimotoa, H. Uyamaa, S. Kobayashib, Synthesis of highperformance green nanocomposites from renewable natural oils, Polymer Degradation and Stability, 95, 8, 1399-1405, (2010).

[7] G. Carbone, C. Putignano. Rough viscoelastic sliding contact: theory and experiments. Physical Review E, 89, 032408, (2014).

[8] Hunter S.C. , The rolling contact of a rigid cylinder with a viscoelastic half space Trans. ASME, Ser. E, J. Appl. Mech. 28, 611-617 (1961).

[9] M. Harrass, K.Friedrich , A.A.Almajid,Tribological behavior of selected engineering polymers under rolling contact, Tribology International,43, 635-646, 2010.

[10] Grosch K. A. , The Relation between the Friction and Visco-Elastic Properties of Rubber, Proceedings of the Royal Society of London. Series A, Mathematical and Physical,274-1356, pp. 21-39, (1963).

[11] Persson B.N.J., Rolling friction for hard cylinder and sphere on viscoelastic solid, Eur. Phys. J. E 33, 327-333 (2010). 
[12] Persson B. N. J., Theory of rubber friction and contact mechanics, J. Chem. Phys. 115, 3840 (2001).

[13] Persson B. N. J., Rubber friction: role of the flash temperature, J. Phys.: Condens. Matter 18 7789, (2006).

[14] Panek C. and Kalker J.J., Three-dimensional Contact of a Rigid Roller Traversing a Viscoelastic Half Space, J. Inst. Maths Applies 26, 299-313, (1980).

[15] J. Padovan, O. Paramadilok, Transient and steady state viscoelastic rolling contact, Comput Struct, 20, 545-553, 1984.

[16] Vollebregt E.A.H., User guide for CONTACT, J.J. Kalker's variational contact model, Technical Report TR09-03, version 1.18, (2011).

[17] Christensen R. M., Theory of viscoelasticity,Academic Press, New York (1971).

[18] J. Padovan. Finite element analysis of steady and transiently moving/rolling nonlinear viscoelastic structure-I. theory. Computers \& Structures, 27(2):249-257, (1987).

[19] J. Padovan, A. Kazempour, F. Tabaddor, and B. Brockman. Alternative formulations of rolling contact problems. Finite Elements in Analysis and Design, 11:275-284, (1992).

[20] L. Nasdala, M. Kaliske, A. Becker, H. Rothert, An efficient viscoelastic formulation for steady-state rolling structures, Computational Mechanics 22, 395-403, (1998).

[21] Le Tallec P., Rahler C., Numerical models of steady rolling for non-linear viscoelastic structures in finite deformations, International Journal for Numerical Methods in Engineering, vol. 37, 1159-1186 (1994).

[22] J. Halaunbrenner, A. Kubisz: ASLE-ASME Lubrication Conf., Chicago (1967) Paper No. 67-Lub-25.

[23] Johnson K.L.J., Contact Mechanics, Cambridge University Press (1985). 
[24] B. A. Krick, Jennifer R. Vail, Bo N. J. Persson, W. G. Sawyer, Optical In Situ Micro Tribometer for Analysis of Real Contact Area for Contact Mechanics, Adhesion, and Sliding Experiments. Tribol Lett. DOI 10.1007/s11249-011-9870-y

[25] B. Lorenz, B.N.J. Persson, S. Dieluweit, and T. Tada, Rubber friction: Comparison of theory with experiment, DOI 10.1140/epje/i2011-111291 Eur. Phys. J. E (2011) 34: 129 THE EUROPEAN PHYSICAL JOURNAL E.

[26] G. Fortunato, V. Ciaravola, A. Furno, B. Lorenz and B N J Persson, General theory of frictional heating with application to rubber friction, Journal of Physics: Condensed Matter, 27, 17, (2015).

[27] C. Putignano, J. Le Rouzic, T. Reddyhoff, G. Carbone, D. Dini, A Theoretical and Experimental Study of Viscoelastic Rolling Contacts Incorporating Thermal Effects, Proceedings of the Institution of Mechanical Engineers, Part J: Journal of Engineering Tribology, vol. 228, no. 10, 1112-1121, (2014).

[28] D. Maugis, Adhesion of Spheres: The JKR-DMT Transition Using a Dugdale Model, Journal of Colloid and Interface Science, 150, 1, (1992).

[29] John D. Ferry, Viscoelastic Properties of Polymers, John Wiley \& Sons, Inc.

[30] Putignano C, Reddyhoff T, Carbone G, Dini D (2013). Experimental Investigation of Viscoelastic Rolling Contacts: A Comparison with Theory. Tribology Letters, vol. 51, p. 105-113, ISSN: 1023-8883, doi: 10.1007/s11249-013-0151-9.

[31] C. Putignano, T. Reddyhoff, D. Dini, C. Carbone, Experimental investigation of viscoelastic rolling contacts: a comparison with theory. Tribology Letters, 51 (1), pp. 105-113, (2013).

[32] I. Goryachevaa, Farshid Sadeghi, Contact characteristics of a rolling/sliding cylinder and a viscoelastic layer bonded to an elastic substrate, 184, 2, 125âa"132, 1995. 
[33] C. Putignano, G. Carbone, D. Dini, Mechanics of Rough Contacts in Elastic and Viscoelastic Thin Layers, Accepted on International Journal of Solids and Structure, doi:10.1016/j.ijsolstr.2015.04.034

[34] Nackenhorst U, The ALE-formulation of bodies in rolling contact Theoretical foundations and finite element approach, Comput Method Appl M, 193, 4299-4322, 2004.

[35] Koumia KE, Chaisea T, Nelias D,Rolling contact of a rigid sphere/sliding of a spherical indenter upon a viscoelastic half-space containing an ellipsoidal inhomogeneity, Journal of the Mechanics and Physics of Solids 80, 1â"'25, 2015.

[36] D. Felhõs, D. Xu1, A. K. Schlarb, K. Váradi, T. Goda, Viscoelastic characterization of an EPDM rubber and finite element simulation of its dry rolling friction, eXPRESS Polymer Letters 2, 3,157-164, 2008 .

[37] Dumitru N. Olaru, Ciprian Stamate, Gheorghe Prisacaru, Rolling Friction in a Micro Tribosystem, Tribol Lett , 35:205-210, 2009 ..

[38] S. Yoneyama, J. Gotoh and M. Takashi, Experimental Analysis of Rolling Contact Stresses in a Viscoelastic Strip, Volume 40, Number 2, 203-210, DOI: 10.1007/BF02325047.

[39] C. Putignano, L. Afferrante, G. Carbone, G. Demelio. A new efficient numerical method for contact mechanics of rough surfaces. International Journal of Solids and Structures, 49 (2), (2012).

[40] C. Putignano, L. Afferrante, G. Carbone , G. Demelio, The influence of the statistical properties of self-affine surfaces in elastic contact: a numerical investigation, Journal of Mechanics and Physics of Solids, 60, 5, 973-982 (2012).

[41] C. Putignano, L. Afferrante, G. Carbone, G. Demelio, A multiscale analysis of elastics contacts between rough surfaces. Tribology International. 64, 148-154, (2013).

[42] C. Putignano, G. Carbone, D. Dini, Mechanics of Rough Contacts in Elastic and Viscoelastic Thin Layers, Accepted on International Journal of Solids and Structure, doi:10.1016/j.ijsolstr.2015.04.034. 
[43] M.L. Williams, R.F. Landel, J.D. Ferry, J. Amer. Chem. Soc., 77:3701, 1955.

[44] Carbone G., Lorenz B., Persson B.N.J. and Wohlers A., Contact mechanics and rubber friction for randomly rough surfaces with anisotropic statistical properties, The European Physiscal Journal E - Soft Matter, 29 (3), 275-284, (2009)

[45] Carbone G., Mangialardi L., Adhesion and friction of an elastic halfspace in contact with a slightly wavy rigid surface, Journal of the Mechanics and Physics of Solids, 52 (6), 1267-1287, 2004.

[46] Persson, B.N.J., Rubber friction: Role of the flash temperature, Journal of Physics Condensed Matter, 18 (32), 7789-7823, (2006)

[47] Lorenz B.; Persson B. N. J.; Dieluweit S., tada T., Rubber friction: Comparison of theory with experiment, EUROPEAN PHYSICAL JOURNAL E 34 (12), 129, doi: 10.1140/epje/i2011-11129-1 (2011).

[48] Carbone G., Mangialardi L.: Analysis of adhesive contact of confined layers by using a Green's function approach, The Journal of the Mechanics and Physics of Solids, 56 (2), 684-706 (2008).

[49] J de Vicente, J R Stokes, and H A Spikes, Rolling and sliding friction in compliant, lubricated contact, Proc. IMechE Vol. 220 Part J: J. Engineering Tribology.

[50] Michael Kessler, Advanced Topics in Characterization of Composites, Trafford Publishing, 2004.

[51] Carbone G., Scaraggi M., Tartaglino U. "Adhesive contact of rough surfaces: comparison between numerical calculations and analytical theories", The European Physiscal Journal E - Soft Matter, 30 (1), 65-74 (2009) 